\title{
Erratum to: On odd rank integral quadratic forms: canonical representatives of projective classes and explicit construction of integral classes with square-free determinant
}

\author{
José María Montesinos-Amilibia
}

Published online: 13 March 2015

(C) Springer-Verlag Italia 2015

\section{Erratum to: RACSAM (2015) 109:199-245 DOI 10.1007/s13398-014-0176-4}

The proof of Corollary 13 of the original article is incorrect. It can be adjusted as follows.

In the statement of Lemma 10 of the original article, the word "diagonal" must be deleted, so as to make the statements of Lemmas 10 and 6 equal. This same Lemma 10 or 6 can be generalized as follows.

Lemma 1 Let $g$ be an integral quadratic form of rank $n \geq 1$, and let $p$ be a prime number such that $p \nmid \operatorname{det} g$. Then, there exist an integral $n \times n$ matrix $T$ such that $T^{t} g T=p E$, where $E$ is integral, and det $T$ is $p^{\frac{n+1}{2}}$ if $n$ is odd and $p^{\frac{n+2}{2}}$ if $n$ is even.

Proof The case $n=2 m-1 \geq 1$ is Lemma 10 of the original article. We only need to prove the case $n=2 m \geq 2$. The result is trivial if $m=1$ by taking $T=\langle p, p\rangle$. The remaining of the proof is exactly parallel to the proof of Lemma 10.

Using Lemma 1 we can adjust the proof of Corollary 13 of the original article as follows

Corollary 1 Let $g$ be an integral quadratic form of rank $n=2 m+1$ and $p$ a prime number such that $p^{2}$ divides $\Delta=\operatorname{det} g$. Then there is an integral $n \times n$ matrix $T$ and a rank $n$ integral quadratic form $E$ such that $\operatorname{det} T=p^{m k}$ and

$$
T^{t} g T=p^{k} E
$$

where $k$ is 1 or 2.

The online version of the original article can be found under doi:10.1007/s13398-014-0176-4.

J. M. Montesinos-Amilibia ( $\square)$

Facultad de Matemáticas, Universidad Complutense, 28040 Madrid, Spain

e-mail: jose_montesinos@mat.ucm.es 
Proof Assume that $g$ has the maximal number $c$ of rows (and columns) divisible by $p$ inside its integral class (say, the first $c$ rows and columns of $g$ ). If $c=1$ then, by Lemma 4 in the original article, the first row and column of $g$ is superdivisible by $p$. Then $T=\langle 1, p, p, \ldots, p\rangle$ has $\operatorname{det} T=p^{2 m}$ and

$$
T^{t} f T=p^{2} E,
$$

where $E$ is integral, which completes the proof in case $c=1$. If $c=n$ the result is trivial. We next assume $1<c<n$. Let $h$ be the $(n-c)$-form obtained by deleting the first $c$ rows and columns of $g$. Then $p \nmid \operatorname{det} h$. Otherwise, by Lemma 4 again, there would be an integral $(n-c) \times(n-c)$ matrix $S$ with det $S= \pm 1$ such that $S^{t} h S$ has a row divisible by $p$. Then, denoting the rank $c$ identity matrix by $I_{c}$, the form

$$
\left(I_{c} \oplus S\right)^{t} g\left(I_{c} \oplus S\right)=\left(\begin{array}{ll}
p A & p C \\
p C^{t} & S^{t} h S
\end{array}\right),
$$

is integrally equivalent to $g$ and has more than $c$ rows divisible by $p$, which is impossible. By Lemma 1 applied to the rank $(n-c)$ form $h$, where $c=2 e$ or $c=2 e+1, e \geq 1$, there is an integral $(n-c) \times(n-c)$ matrix $R$ such that det $R=p^{m-e+1}$ and $R^{t} h R=p E_{1}$, where $E_{1}$ is integral. Set $T=I_{c} \oplus R$. Then $\operatorname{det} T=p^{m-e+1}, m \geq m-e+1$, and

$$
T^{t} g T=p E,
$$

where $E$ is integral. This completes the proof of Corollary 13.

The fact that all integral, ternary quadratic zero-forms are commensurable to each other (Theorem 11 in the original article) was essentially proved by Bianchi in [1].

\section{Reference}

1. Bianchi, L.: Sul gruppo automorfo delle forme ternarie quadratiche suscettibili di rappresentare lo zero. Rend. della R. Accad. dei Lincei 21, 305-315 (1912) 